PROCEEDINGS OF THE

AMERICAN MATHEMATICAL SOCIETY

Volume 128, Number 8 , Pages $2421-2430$

S 0002-9939(99)05281-8

Article electronically published on December 7, 1999

\title{
PERIODIC HYPERFUNCTIONS AND FOURIER SERIES
}

\author{
SOON-YEONG CHUNG, DOHAN KIM, AND EUN GU LEE
}

(Communicated by Christopher D. Sogge)

\begin{abstract}
Every periodic hyperfunction is a bounded hyperfunction and can be represented as an infinite sum of derivatives of bounded continuous periodic functions. Also, Fourier coefficients $c_{\alpha}$ of periodic hyperfunctions are of infraexponential growth in $\mathbb{R}^{n}$, i.e., $c_{\alpha}<C_{\epsilon} e^{\epsilon|\alpha|}$ for every $\epsilon>0$ and every $\alpha \epsilon$ $\mathbb{Z}^{n}$. This is a natural generalization of the polynomial growth of the Fourier coefficients of distributions.

To show these we introduce the space $\mathcal{B}_{L^{p}}$ of hyperfunctions of $L^{p}$ growth which generalizes the space $\mathcal{D}_{L^{p}}^{\prime}$ of distributions of $L^{p}$ growth and represent generalized functions as the initial values of smooth solutions of the heat equation.
\end{abstract}

\section{INTRODUCTION}

In this paper we show that the following results:

(i) Every periodic hyperfunction is a bounded hyperfunction.

(ii) Every periodic hyperfunction can be represented as an infinite sum of derivatives of bounded continuous periodic functions.

(iii) Fourier coefficients $c_{\alpha}$ of periodic hyperfunctions are of infra-exponential growth in $\mathbb{R}^{n}$, i.e., $\left|c_{\alpha}\right|<C e^{\epsilon|\alpha|}$ for every $\epsilon>0$. Our result contains the result of Sato and Helgason which deals with the case of $\mathbb{R}^{1}$ (see $[\mathrm{Sa},[\mathrm{He}]$ ).

These results are natural generalization of the well known results in distributions and ultradistributions. See $[\mathrm{S}], \mathrm{G}], \mathrm{GG}]$ for details.

In the proof of these results we use the heat kernel method and introduce the space $\mathcal{B}_{L^{p}}$ of Sato hyperfunctions of $L^{p}$ growth which generalizes the space $\mathcal{D}_{L^{p}}^{\prime}$ of Schwartz distributions of $L^{p}$ growth. This "heat kernel" method, which represents the above generalized functions as the initial values of smooth solutions of heat equations as in Matsuzawa [M], Kim-Chung-Kim [KCK] and Chung-Kim [CK], can overcome difficulties due to the sheaf theoretical definition of the hyperfunctions when we are dealing with the analytical and global properties in the theory of hyperfunctions. Applying this idea we relate the periodic hyperfunctions to the periodicity of its defining function. Note that also in the case of distributions periodicity in terms of the defining function for the distributions and the original definition for periodic distributions coincide naturally. See (2.1) for the definition of the defining function.

Received by the editors June 16, 1998 and, in revised form, September 24, 1998.

1991 Mathematics Subject Classification. Primary 46F15, 35K05, 42B05.

Key words and phrases. Hyperfunction, periodic, Fourier series.

Partially supported by BSRI and GARC-KOSEF. 
In Section 2 using this heat kernel method we state a structure theorem for distributions and Fourier hyperfunctions in $\mathrm{M}$ and $\mathrm{KCK}$ respectively. In Section 3 we also introduce the space $\mathcal{B}_{L^{p}}$ of Sato hyperfunctions of $L^{p}$ growth and give a structure theorem for this space. In Section 4 we define periodicity for hyperfunctions $u$ in terms of its collection of restrictions $u_{j}$ to $\Omega_{j}$ by following A. Martineau's approach in Theorem 2.3 and its following remark. We relate the definition of the periodic hyperfunctions and periodicity of its defining function and show that every periodic hyperfunction is a Fourier hyperfunction and furthermore a bounded hyperfunction, which is parallel to the fact that every periodic distribution is a tempered distribution and furthermore, a bounded distribution. Finally, we show that Fourier coefficients $c_{\alpha}$ of periodic hyperfunctions are of infra-exponential growth, i.e., for every $\epsilon>0 c_{\alpha}<C e^{\epsilon|\alpha|}$, which is a natural generalization of polynomial growth of the Fourier coefficients of distributions and of Gorbačuk's result for the case of ultradistributions in G], GG].

\section{Generalized functions as initial VAlues of SOlutions OF THE HEAT EQUATION}

We first briefly introduce analytic functionals, hyperfunctions and Fourier hyperfunctions. See $[\mathrm{H}],[\mathrm{KCK}],[\mathrm{M}]$ for more details.

Definition 2.1. Let $K \subset \mathbb{R}^{n}$ be a compact set. Then $A(K)$ is the space of all real analytic functions in some neighborhood of $K$ with the seminorms

$$
\sup _{\substack{\alpha \in K \\ \alpha}}\left|\partial^{\alpha} \varphi(x)\right| / h^{|\alpha|} \alpha !,
$$

where we use the multi-index notations: $|\alpha|=\alpha_{1}+\cdots+\alpha_{n}$ for $\alpha=\left(\alpha_{1}, \ldots, \alpha_{n}\right) \in$ $\mathbb{N}_{0}^{n}$ where $\mathbb{N}_{0}$ is the set of non-negative integers and $\partial^{\alpha}=\partial_{1}^{\alpha_{1}} \cdots \partial_{n}^{\alpha_{n}}, \partial_{j}=\partial / \partial x_{j}$.

We denote by $A^{\prime}(K)$ the strong dual space of $A(K)$ and call its element an analytic functional carried by $K$.

We set $A^{\prime}\left(\mathbb{R}^{n}\right)=\bigcup_{K} A^{\prime}(K)$ and the support of $u \in A^{\prime}\left(\mathbb{R}^{n}\right)$ is the smallest compact set $K \subset \mathbb{R}^{n}$ such that $u \in A^{\prime}(K)$.

We are in a position to define the space $\mathcal{B}$ of hyperfunctions following A. Martineau as in Hörmander $[\mathrm{H}]$ and to state the localization theorem.

Definition 2.2. Let $\Omega$ be a bounded open set in $\mathbb{R}^{n}$. Then the space $\mathcal{B}(\Omega)$ of hyperfunctions is defined by $\mathcal{B}(\Omega)=A^{\prime}(\bar{\Omega}) / A^{\prime}(\partial \Omega)$.

Theorem 2.3. Let $\Omega_{j}, j=1,2, \ldots$, be bounded open subsets of $\mathbb{R}^{n}$ such that $\Omega=\bigcup_{i=1}^{\infty} \Omega_{j}$. If $u_{j} \in \mathcal{B}\left(\Omega_{j}\right)$ and for all $i, j$ we have $u_{i}=u_{j}$ in $\Omega_{i} \cap \Omega_{j}$ (that $i s$, supp $\left.\left(u_{i}-u_{j}\right) \cap \Omega_{i} \cap \Omega_{j}=\emptyset\right)$, then there is a unique $u \in \mathcal{B}(\Omega)$ such that the restriction of $u$ to $\Omega_{j}$ is equal to $u_{j}$.

Remark 2.4. It follows from the above theorem that a hyperfunction $u \in B\left(\mathbb{R}^{n}\right)$ can be regarded as a collection of $u_{j} \in \mathcal{A}^{\prime}\left(\overline{\Omega_{j}}\right)$ with $\mathbb{R}^{n}=\bigcup_{j} \Omega_{j}$, and we write $u=\left(u_{j}\right)$.

We now introduce a real version of the Fourier hyperfunctions. 
Definition $2.5([\overline{K C K}])$. We denote by $\mathcal{F}$ the Sato space of all infinitely differentiable functions $\varphi$ in $\mathbb{R}^{n}$ such that the seminorms

$$
|\varphi|_{h, k}=\sup _{\substack{x \in \mathbb{R}^{n} \\ \alpha \in \mathbb{N}_{0}^{n}}} \frac{\left|\partial^{\alpha} \varphi(x)\right| \exp k|x|}{h^{|\alpha|} \alpha !}<\infty
$$

are finite for some $h, k>0$ and these seminorms define its topology.

We denote by $\mathcal{F}^{\prime}$ the strong dual of $\mathcal{F}$ and call its elements Fourier hyperfunctions.

We denote by $E(x, t)$ the $n$-dimensional heat kernel

$$
E(x, t)= \begin{cases}(4 \pi t)^{-n / 2} \exp \left(-|x|^{2} / 4 t\right), & t>0, \\ 0, & t \leq 0 .\end{cases}
$$

Note that $E(x, t)$ belongs to the Sato space $\mathcal{F}$ for each $t>0$.

Definition 2.6. For every $u \in \mathcal{F}^{\prime}$

$$
U(x, t)=u_{y}(E(x-y, t))
$$

is well defined in $\mathbb{R}_{+}^{n+1}=\left\{(x, t) \mid x \in \mathbb{R}^{n}, t>0\right\}$ and called the defining function of $u$.

We now represent distributions, hyperfunctions as in Matsuzawa [M] and Fourier hyperfunctions as in Kim-Chung-Kim [KCK], as the initial values of smooth solutions of the heat equation.

Theorem $2.7(\underline{\mathrm{M}}])$. (i) Let $u \in \mathcal{D}^{\prime}\left(\mathbb{R}^{n}\right)$. Then there is a defining function $U(x, t)$ $\in C^{\infty}\left(\mathbb{R}_{+}^{n+1}\right)$ satisfying the following conditions:

$$
(\partial / \partial t-\Delta) U(x, t)=0 \quad \text { in } \mathbb{R}_{+}^{n+1} .
$$

For any compact set $K \subset \mathbb{R}^{n}$ there exist positive constants $N=N(K)$ and $C_{K}$ such that

$$
|U(x, t)| \leq C_{K} t^{-N}, \quad t>0, x \in K,
$$

and $U(\cdot, t) \rightarrow u$ as $t \rightarrow 0^{+}$in the sense that for every $\varphi \in C_{c}^{\infty}$

$$
u(\varphi)=\lim _{t \rightarrow 0^{+}} \int U(x, t) \varphi(x) d x .
$$

Conversely, let $U(x, t) \in C^{\infty}\left(\mathbb{R}_{+}^{n+1}\right)$ satisfy (2.2) and (2.3). Then there exists a unique $u \in \mathcal{D}^{\prime}\left(\mathbb{R}^{n}\right)$ satisfying (2.4).

(ii) Let $u \in \mathcal{B}\left(\mathbb{R}^{n}\right)$. Then there is a defining function $U(x, t) \in C^{\infty}\left(\mathbb{R}_{+}^{n+1}\right)$ satisfying the heat equation (2.2) and the following conditions: For every compact subset $K \subset \mathbb{R}^{n}$ and for every $\epsilon>0$ there exists a constant $C_{\epsilon, K}>0$ such that

$$
|U(x, t)| \leq C_{\epsilon, K} \exp (\epsilon / t), \quad t>0, x \in K,
$$

and

$$
U(\cdot t) \rightarrow u \quad \text { as } t \rightarrow 0^{+}
$$

in the sense that $U(x, t)-U_{j}(x, t) \Longrightarrow 0$ as $t \rightarrow 0^{+}$in $\Omega_{j}, j=1,2, \ldots$, where $\Longrightarrow$ means weak uniform convergence in $\Omega_{j}$ and $U_{j}$ is the defining function of $u_{j} \in A^{\prime}\left(\bar{\Omega}_{j}\right)$ as in (2.1) and $u=\left(u_{j}\right) \in \mathcal{B}\left(\mathbb{R}^{n}\right)$ with $\mathbb{R}^{n}=\bigcup_{j} \Omega_{j}$.

Conversely, let $U(x, t) \in C^{\infty}\left(\mathbb{R}_{+}^{n+1}\right)$ satisfy (2.2) and (2.5). Then there exists a unique $u \in \mathcal{B}\left(\mathbb{R}^{n}\right)$ satisfying (2.6). 
Remark 2.8 ([KCK] $)$. If we replace the growth condition (2.5) by

$$
|U(x, t)| \leq C_{\epsilon} \exp [\epsilon(|x|+1 / t)],
$$

then we obtain the case for Fourier hyperfunctions.

\section{Hyperfunctions OF $L^{p}$ GROWTH}

We first introduce the new space $\mathcal{B}_{L^{p}}$ of hyperfunctions of $L^{p}$ growth which is a natural generalization of $\mathcal{D}_{L^{p}}^{\prime}$ for hyperfunctions and will be used to characterize the periodic hyperfunctions. We also represent these hyperfunctions as the initial values of solutions of the heat equation.

Definition 3.1. We denote by $\mathcal{A}_{L^{q}}(1 \leq q<\infty)$ the space of all functions $\varphi \in$ $C^{\infty}\left(\mathbb{R}^{n}\right)$ satisfying

$$
\|\varphi\|_{L^{q}, h}=\sup _{\alpha} \frac{\left\|\partial^{\alpha} \varphi\right\|_{L^{q}}}{h^{|\alpha|} \alpha !}<\infty
$$

for some constant $h>0$. We say that $\varphi_{j} \rightarrow 0$ in $\mathcal{A}_{L^{q}}$ as $j \rightarrow \infty$ if there is a positive constant $h$ such that

$$
\sup _{\alpha} \frac{\left\|\partial^{\alpha} \varphi_{j}\right\|_{L^{q}}}{h^{|\alpha|} \alpha !} \rightarrow 0 \quad \text { as } j \rightarrow \infty .
$$

We denote by $\mathcal{B}_{L^{p}}(1<p \leq \infty)$ the dual of $\mathcal{A}_{L^{q}}$, where $1 / p+1 / q=1$. In particular, every element in $\mathcal{B}_{L^{\infty}}$ is called a bounded hyperfunction. Note that every element in $\mathcal{D}_{L^{\infty}}^{\prime}$ is called a bounded distribution.

It is easy to see the following topological inclusions:

$$
\mathcal{F} \hookrightarrow \mathcal{A}_{L^{q}}(1 \leq q<\infty), \quad \mathcal{B}_{L^{p}} \hookrightarrow \mathcal{F}^{\prime}(1<p \leq \infty) .
$$

We will prove the structure theorem for $\mathcal{B}_{L^{p}}(1<p \leq \infty)$. We need the following two lemmas to prove the main theorem.

Lemma $3.2([\mathrm{M}])$. For any $h>0$ and $\epsilon>0$ there exist functions $v(t) \in C_{c}^{\infty}([0, \epsilon])$ and an ultradifferential operator $P(d / d t)$ such that

$$
\begin{gathered}
\left|v^{(k)}(t)\right| \leq C h^{-k} k !^{2}, \quad k=0,1, \cdots, \\
|v(t)| \leq C \exp (-h / t), \quad 0<t<\infty, \\
P(d / d t)=\sum_{k=0}^{\infty} a_{k}(d / d t)^{k},\left|a_{k}\right| \leq C_{1} h_{1}^{k} / k !^{2}, \quad 0<h_{1}<h, \\
P(d / d t) v(t)=\delta+w(t),
\end{gathered}
$$

where $w(t) \in C_{c}^{\infty}([\epsilon / 2, \epsilon])$.

Lemma 3.3. For every $\varphi \in \mathcal{A}_{L^{q}}(1 \leq q<+\infty)$, let

$$
\varphi_{t}(x)=\int E(x-y, t) \varphi(y) d y, \quad t>0 .
$$

Then $\varphi_{t} \in \mathcal{A}_{L^{q}}$ and $\varphi_{t} \rightarrow \varphi$ in $\mathcal{A}_{L^{q}}$ as $t \rightarrow 0^{+}$. 
Proof. Let $\varphi \in \mathcal{A}_{L^{q}}$. Then, clearly $\varphi_{t} \in \mathcal{A}_{L^{q}}$. Also, by the same method as in Kim-Chung-Kim [KCK Theorem 1.3] we can easily show that for every $\epsilon>0$ there exists a constant $\delta>0$ such that, for $0<t<\delta$,

$$
\frac{\left\|\partial^{\alpha} \varphi_{t}-\partial^{\alpha} \varphi\right\|_{L^{q}}}{h^{|\alpha|} \alpha !}<\epsilon
$$

for all $\alpha$ where $\delta$ is independent of $\alpha$. Thus we obtain that $\varphi_{t}(x) \rightarrow \varphi(x)$ in $\mathcal{A}_{L^{q}}$ as $t \rightarrow 0^{+}$.

Let $u \in \mathcal{B}_{L^{p}}$. Then the defining function $U(x, t)=u_{y}(E(x-y, t)),(x, t) \in \mathbb{R}_{+}^{n+1}$, is a well defined $C^{\infty}$ function, since $E(x-\cdot, t)$ belongs to $\mathcal{A}_{L^{q}}(1 / p+1 / q=1)$ for each $(x, t) \in \mathbb{R}_{+}^{n+1}$.

Theorem 3.4. A hyperfunction $u$ belongs to $\mathcal{B}_{L^{p}}(1<p \leq \infty)$ if and only if $u$ can be written as

$$
u=P(-\Delta) g(x)+h(x)
$$

where $P(-\Delta)$ is given in Lemma 3.2 and $g$ and $h$ are bounded continuous functions belonging to $L^{p}$.

Proof. Sufficiency: If $\varphi \in \mathcal{A}_{L^{q}}(1 \leq q<\infty)$ with $\|\varphi\|_{L^{q}, h}<\infty(1 / p+1 / q=1)$, then $P(-\Delta) \varphi \in L^{q}$ and for $\epsilon<2 / n h^{2}$,

$$
\begin{aligned}
\|P(-\Delta) \varphi\|_{L^{q}} & \leq \sum_{k=0}^{\infty}\left|a_{k}\right|\left\|(-\Delta)^{k} \varphi\right\|_{L^{q}} \\
& \leq \sum_{k=0}^{\infty} \frac{\left(\epsilon n h^{2}\right)^{k}(2 k) !}{k !^{2}}\|\varphi\|_{L^{q}, h} \\
& \leq C\|\varphi\|_{L^{q}, h}
\end{aligned}
$$

for some constant $C>0$. Therefore, we can easily obtain that $u$ is a bounded linear functional on $\mathcal{A}_{L^{q}}$.

Necessity: We first prove that $u * \varphi \in L^{p}$ for all $\varphi \in \mathcal{F}$. We denote by $B$ the unit disc with respect to the $L^{q}$ norm, i.e., $B=\left\{\psi \in \mathcal{F}:\|\psi\|_{L^{q}} \leq 1\right\}$. Then $B$ is dense in the unit ball of $L^{q}$. Consider $\varphi * \psi$ for fixed $\varphi$ and $\psi \in B$. Then it follows that $(u * \varphi)(\psi)=u(\check{\varphi} * \psi)$ is bounded. Therefore, $u * \varphi$ is a bounded linear functional on $\mathcal{F}$ by topology induced by $L^{q}$. Thus $u * \varphi \in L^{p}$.

By Lemma 3.2 there exist functions $v, w \in C_{c}^{\infty}(\mathbb{R})$ such that $P(d / d t) v+w=\delta$. Define

$$
V(x, t)=\int_{0}^{\infty} E(x, t+s) v(s) d s \quad \text { and } \quad W(x, t)=\int_{0}^{\infty} E(x, t+s) w(s) d s .
$$

Then it follows that $V(x, t)$ and $W(x, t)$ are contained in $\mathcal{F}$ for fixed $t>0$ and in $C^{\infty}\left(\mathbb{R}_{+}^{n+1}\right)$.

Therefore, we can see that $u *_{x} V(x, t)$ and $u *_{x} W(x, t)$ belong to $L^{p}$ for every fixed $t>0$. Moreover, we have

$$
\begin{gathered}
\left(\partial_{t}-\Delta\right) u *_{x} V=0, \quad\left(\partial_{t}-\Delta\right) u *_{x} W=0, \\
P(-\Delta) u *_{x} V+u *_{x} W=u *_{x} E .
\end{gathered}
$$

Then by the well known theorem in Widder [W] there exist functions $g(x)$ and $h(x)$ in $L^{p}$ such that

$$
u * V(x, t)=g(x) * E(x, t), \quad u * W(x, t)=h(x) * E(x, t) .
$$


In fact, it follows from $u * V(x, 0)=g(x)$ and $u * W(x, 0)=h(x)$ that $g(x)$ and $h(x)$ are also bounded continuous functions on $\mathbb{R}^{n}$.

Therefore, by the uniqueness of defining function $U(x, t)=u_{y}(E(x-y, t))$ of $u$ we have $u=P(-\Delta) g+h$, which completes the proof.

Theorem 3.5. Let $u \in \mathcal{B}_{L^{p}}$. Then the defining function $U(x, t)$ of $u$ belongs to $C^{\infty}\left(\mathbb{R}_{+}^{n+1}\right)$ and satisfies the following:

$$
\left(\partial_{t}-\Delta\right) U(x, t)=0 \text { in } \mathbb{R}_{+}^{n+1} .
$$

For every $\epsilon>0$ there exists a constant $C>0$ such that

$$
\|U(x, t)\|_{L^{p}\left(\mathbb{R}_{x}^{n}\right)} \leq C e^{\epsilon / t} \quad \text { in } \mathbb{R}_{+}^{n+1},
$$

and $U(\cdot, t) \rightarrow u$ as $t \rightarrow 0^{+}$in the sense that

$$
u(\phi)=\lim _{t \rightarrow 0^{+}} \int_{\mathbb{R}^{n}} U(x, t) \phi(x) d x, \quad \phi \in \mathcal{A}_{L^{q}} .
$$

Conversely, every $C^{\infty}$ function defined in $\mathbb{R}_{+}^{n+1}$ satisfying condition (3.2) and (3.3) can be written as

$$
U(x, t)=u_{y}(E(x-y, t)) \quad \text { in } \mathbb{R}_{+}^{n+1}
$$

with a unique element $u \in \mathcal{B}_{L^{p}}$.

Proof. Let $u \in \mathcal{B}_{L^{p}}$. Then it follows from Lemma 3.3 that

$$
\int U(x, t) \varphi(x) d x=u_{y}\left(\varphi_{t}(x)\right)
$$

for every $\varphi \in \mathcal{A}_{L^{q}}$, which gives (3.2).

Since $u \in \mathcal{B}_{L^{p}}$, we can write $u=P(-\Delta) g+h$ where $g$ and $h$ are bounded continuous functions belonging to $L^{p}$. Then we have

$$
\begin{aligned}
\|U(x, t)\|_{L^{p}\left(\mathbb{R}_{x}^{n}\right)} & =\|u * E\|_{L^{p}} \\
& \leq\|P(-\Delta) g * E\|_{L^{p}}+\|h * E\|_{L^{p}} \\
& =\|g * P(-\Delta) E\|_{L^{p}}+\|h * E\|_{L^{p}} \\
& \leq\|g\|_{L^{p}} \sum_{k=0}^{\infty}\left\|a_{k}(-\Delta)^{k} E\right\|_{L^{1}}+\|h\|_{L^{p}}\|E\|_{L^{1}} .
\end{aligned}
$$

Applying the following estimate in [M, p.622]:

$$
\left|\partial_{x}^{\alpha} E(x, t)\right| \leq C^{|\alpha|} t^{-(n+|\alpha|) / 2} \alpha !^{1 / 2} \exp \left(-|x|^{2} / 8 t\right), \quad(x, t) \in \mathbb{R}_{+}^{n+1},
$$

we can obtain that for some $C>0$

$$
\left\|\partial^{\alpha} E(x, t)\right\|_{L^{1}\left(\mathbb{R}_{x}^{n}\right)} \leq C^{|\alpha|} t^{-(|\alpha|+n) / 2} \alpha !^{\frac{1}{2}}\left\|\exp \left(-\frac{|x|^{2}}{8 t}\right)\right\|_{L^{1}\left(\mathbb{R}_{x}^{n}\right)^{\prime}} .
$$

Therefore, it follows that for every $\epsilon=2 h C^{2}>0$

$$
\|U(x, t)\|_{L^{p}} \leq C \sum_{k=0}^{\infty} \frac{h^{k}}{k !^{2}} C^{2 k} t^{-k}(2 k) !^{1 / 2} \leq C \sum_{k=0}^{\infty} \frac{\left(2 h C^{2}\right)^{k} t^{-k}}{k !}=C e^{\epsilon / t}
$$

in $\mathbb{R}_{+}^{n+1}$.

We now prove the converse. By (3.3) we can define the convolution of $v$ or $w$ given in Lemma 3.3 and $U(x, t)$. Therefore we can prove the converse by the same method as in Theorem 3.4. 
Remark. In Theorem 3.4 we can obtain that $u * \varphi \in L^{p}$ for every $\varphi \in \mathcal{F}$ if and only if $u \in \mathcal{B}_{L^{p}}$.

\section{Periodic hyperfunctions}

In this section we give a definition of periodic hyperfunctions $u$ in terms of the collection of analytic functionals with compact support $u_{j} \in \mathcal{A}^{\prime}\left(\Omega_{j}\right)$ as in Remark 2.4 along lines proposed by Martineau. Also, we relate this definition of periodic hyperfunctions and the periodicity of the defining function $U$ of $u$ as in Theorem 4.2 .

We now give a natural definition of periodic hyperfunctions.

Definition 4.1. A hyperfunction $u=\left(u_{j}\right)_{j \in \mathbb{Z}} \in B\left(\mathbb{R}^{n}\right)$, where $u_{j} \in \mathcal{A}^{\prime}\left(\overline{\Omega_{j}}\right)$ with $\mathbb{R}^{n}=\bigcup_{j} \Omega_{j}$ is periodic if $\tau_{\alpha} u=\left(\tau_{\alpha} u_{j}\right)=u$ for all $\alpha \in \mathbb{Z}^{n}$. Here, $\tau_{\alpha} u_{j} \in \mathcal{A}^{\prime}\left(\tau_{\alpha} \overline{\Omega_{j}}\right)$ with $\tau_{\alpha} \Omega_{j}=\left\{x+\alpha \mid x \in \Omega_{j}\right\}$.

From this definition we can easily obtain the following

Theorem 4.2. If a hyperfunction $u$ is periodic, then the defining function $U(x, t)$ of $u$ is also periodic.

Proof. Let $U_{j}(x, t)=u_{j} * E(x, t)$ be the defining function of $u_{j}$ and $V_{j}(x, t)=$ $\tau_{\alpha} u_{j} * E(x, t)$ be the defining function of $\tau_{\alpha} u_{j}$. Then we have $V_{j}(x, t)=U_{j}(x+\alpha, t)$. In fact,

$$
V_{j}(x, t)=u_{j}(E(x-(\cdot-\alpha), t))=u_{j} * E(x+\alpha, t)=U_{j}(x+\alpha, t) .
$$

Therefore, the defining function of $\tau_{\alpha} u$ is $U(x+\alpha, t)$. It follows from $\tau_{\alpha} u=u$ that $U(x+\alpha, t)=U(x, t)$ for all $\alpha \in \mathbb{Z}^{n}$.

Making use of the periodicity of the defining function $U$ of a periodic hyperfunction $u$ we now prove the following

Theorem 4.3. Every periodic hyperfunction is a bounded hyperfunction.

Proof. Let $U(x, t)$ be a defining function of $u$ which is periodic with respect to $x$. It follows from Theorem 2.7 that for any compact subset $K$ of $\mathbb{R}^{n}$ and $\epsilon>0$, we have

$$
|U(x, t)| \leq C_{\epsilon, K} e^{\epsilon / t}, \quad(x, t) \in \mathbb{R}_{+}^{n+1} .
$$

But since $U(x, t)$ is periodic, we can choose $C_{\epsilon, K}=C_{\epsilon}$ independent of $K$. Therefore, by Theorem 3.5 we have $u \in \mathcal{B}_{L^{\infty}}$.

Remark. It follows from Theorem 4.3 that every periodic hyperfunction $u$ is a periodic Fourier hyperfunction, i.e., $u$ is periodic in $\mathcal{F}^{\prime}$. Therefore, we obtain that $\tau_{\alpha} u(\varphi)=u(\varphi)$ for all $\alpha \in \mathbb{Z}^{n}, \varphi \in \mathcal{F}$.

We now show that a periodic hyperfunction can be expanded as a Fourier series with coefficients of infra-exponential growth, which generalizes the results of Gorbačuk's [G], GG] and the similar result for distributions.

Theorem 4.4. The trigonometric series $u=\sum_{\alpha \in \mathbb{Z}^{n}} c_{\alpha} e^{2 \pi i \alpha \cdot x}$ is the Fourier series of a periodic hyperfunction, i.e., $\tau_{\alpha} u=u$ for all $\alpha \in \mathbb{Z}^{n}$ if and only if for every $\epsilon>0$ there exists a constant $C>0$ such that $\left|c_{\alpha}\right| \leq C e^{\epsilon|\alpha|}$ for every $\alpha \in \mathbb{Z}^{n}$, where $|\alpha|=\left|\alpha_{1}\right|+\cdots+\left|\alpha_{n}\right|$. 
Proof. Necessity: It follows from Theorem 3.4 and Theorem 4.3 that $u$ can be written as $u=P(-\Delta) f+g$ where $P(-\Delta)$ is given in Lemma 3.2 and $f$ and $g$ are bounded continuous functions. We can easily obtain that $f$ and $g$ are periodic functions as in the proof of Theorem 3.4. Accordingly, expanding $f$ and $g$ as Fourier series we write

$$
f=\sum_{\alpha \in \mathbb{Z}^{n}} \hat{f}(2 \pi \alpha) e^{2 \pi i \alpha \cdot x}, \quad g=\sum_{\alpha \in \mathbb{Z}^{n}} \hat{g}(2 \pi \alpha) e^{2 \pi i \alpha \cdot x} .
$$

Therefore, we have the following identity:

$$
P(-\Delta) f+g=\sum_{\alpha \in \mathbb{Z}^{n}}\left(P\left(4 \pi^{2} \alpha^{2}\right) \hat{f}(2 \pi \alpha)+\hat{g}(2 \pi \alpha)\right) e^{2 \pi i \alpha \cdot x},
$$

where $\alpha^{2}=\alpha_{1}^{2}+\cdots+\alpha_{n}^{2}$. Writing

$$
c_{\alpha}=P\left(4 \pi^{2} \alpha^{2}\right) \hat{f}(2 \pi \alpha)+\hat{g}(2 \pi \alpha)
$$

we finally obtain that, for every $\epsilon=2 \pi \sqrt{h}$,

$$
\begin{aligned}
\left|c_{\alpha}\right| & \leq\|f\|_{L^{\infty}} \sum_{k=0}^{\infty}\left|a_{k}\right|\left|\left(4 \pi^{2} \alpha^{2}\right)^{k}\right|+\|g\|_{L^{\infty}} \\
& \leq C \sum_{k=0}^{\infty} \frac{\left(4 \pi^{2} h\right)^{k}}{k !^{2}}|\alpha|^{2 k} \\
& \leq C e^{2 \epsilon|\alpha|} .
\end{aligned}
$$

Sufficiency: If the growth condition for the coefficients $c_{\alpha}$ is satisfied, we can define a linear form $u$ on $\mathcal{A}_{L^{1}}$ by

$$
u(\varphi)=\sum_{\alpha \in \mathbb{Z}^{n}}\left(c_{\alpha} e^{2 \pi i \alpha \cdot x}\right)(\varphi), \quad \varphi \in \mathcal{A}_{L^{1}}
$$

Then $u$ is continuous on $\mathcal{A}_{L^{1}}$. In fact, for all $\varphi \in \mathcal{A}_{L^{1}}$ with $\|\varphi\|_{L^{1}, h}<\infty$ there exist constants $\epsilon, C$ with $\epsilon<1 / 2 h n$ such that

$$
\begin{aligned}
u(\varphi) & =\sum_{\alpha \in \mathbb{Z}^{n}}\left|c_{\alpha}\right||\hat{\varphi}(-2 \pi \alpha)| \\
& \leq C_{1} \sum_{\alpha \in \mathbb{Z}^{n}} \sum_{k=0}^{\infty} \frac{(\epsilon|\alpha|)^{k}}{k !}|\hat{\varphi}(-2 \pi \alpha)| \\
& \leq C_{2} \sum_{k=0}^{\infty} \frac{(\epsilon)^{k}}{k !} \sum_{\alpha \in \mathbb{Z}^{n}} \frac{1}{|\alpha|^{n+1}} \sum_{|\beta|=k+n+1} \frac{(k+n+1) !}{\beta !}\left\|\partial^{\beta} \varphi\right\|_{L^{1}} \\
& \leq C_{3} \sum_{k=0}^{\infty}(2 \epsilon h n)^{k}\|\varphi\|_{L^{1}, h} \\
& \leq C\|\varphi\|_{L^{1}, h} .
\end{aligned}
$$

Remark. One dimensional version of the above Theorem 4.4 was given by Sato in $[\mathrm{S}]$ and Helgason [He, Lemma 4.25] by using a quite different method from our heat kernel method. 
We finally estimate the partial sum of Fourier series.

Theorem 4.5. Let $u$ be a periodic hyperfunction. Then for every $\epsilon>0$ there exists a constant $C>0$ such that

$$
\left|s_{m}(u, x)\right|=\sum_{|\alpha| \leq m}\left|c_{\alpha} e^{2 \pi i \alpha \cdot x}\right| \leq C e^{\epsilon m} .
$$

Proof. Also, if $s_{m}(u, x)=\sum_{|\alpha| \leq m} c_{\alpha} e^{2 \pi i \alpha \cdot x}$, then

$$
\begin{aligned}
\left|s_{m}(u, x)\right| & \leq \sum_{|\alpha| \leq m}\left|c_{\alpha}\right| \\
& \leq C \sum_{|\alpha| \leq m} \sum_{k=0}^{\infty} \frac{\epsilon^{k}|\alpha|^{k}}{k !} \\
& =C \sum_{k=0}^{\infty} \sum_{|\alpha| \leq m} \frac{1}{|\alpha|^{2}} \frac{(k+2)(k+1) \epsilon^{k}|\alpha|^{k+2}}{(k+2) !} \\
& \leq C \sum_{k=0}^{\infty} \frac{m^{k+2}(2 \epsilon)^{k+2}}{(k+2) !} \sum_{|\alpha| \leq m} \frac{\epsilon^{-2}}{|\alpha|^{2}} \\
& \leq C^{\prime} \sum_{k=0}^{\infty} \frac{(2 \epsilon)^{k+2} m^{k+2}}{(k+2) !} \\
& \leq C^{\prime} e^{2 \epsilon m} .
\end{aligned}
$$

\section{ACKNOWLEDGEMENT}

We would like to express our gratitude to Professor M. Morimoto for his suggestion of Definition 4.1 and his interest in our paper.

\section{REFERENCES}

[CCK1] J. Chung, S.-Y. Chung and D. Kim, Une caractérisation de l'espace de Schwartz, C. R. Acad. Sci. Paris Sér. I Math. 316 (1993), 23-25. MR 93m:46040

[CCK2] , A characterization for Fourier hyperfunctions, Publ. RIMS, Kyoto Univ. 30 (1994), 203-208. MR 96m:46066

[CCK3] _ Positive definite hyperfunctions, Nagoya Math. J. 140 (1995), 139-149. MR 96m:46075

[CK] S.-Y. Chung and D. Kim, Representation of quasianalytic ultradistributions, Ark. Mat. 31 (1993), 51-60. MR 94e:46069

[GS] I. M. Gelfand and G. E. Shilov, Generalized functions I, II and IV, Academic Press, New York, 1968. MR 55:8786a MR 55:8786b MR 55:8786c

[G] V. I. Gorbačuk, On Fourier series of periodic ultradistributions, Ukrainian Math. J. (2) 34 (1982), 144-150.

[GG] V. I. Gorbačuk and M. L. Gorbačuk, Trigonometric series and generalized functions, Dokl. Akad. Nauk SSSR (4) 257 (1981), 799-804. MR 82d:42008

[He] S. Helgason, Topics in harmonic analysis on homogeneous spaces, Birkhäuser, Boston, 1981. MR 83g:43009

$[\mathrm{H}]$ L. Hörmander, The analysis of linear partial differential operators I, Springer-Verlag, Berlin-New York, 1983. |MR 85g:35002a

[Kt] Y. Katznelson, An introduction to harmonic analysis, Dover Publ., New York, 1976. MR 54:10976 
$[\mathrm{KCK}]$ K. H. Kim, S.-Y. Chung and D. Kim, Fourier hyperfunctions as the boundary values of smooth solutions of heat equations, Publ. RIMS, Kyoto Univ. 29 (1993), 289-300. MR 94m: 46076

[M] T. Matsuzawa, A calculus approach to hyperfunctions II, Trans. Amer. Math. Soc. 313 (1990), 619-654. MR 90g:46062

[Sa] M. Sato, Theory of hyperfunctions, Sugaku 10 (1958), 1-27 (Japanese). MR 24:A2237

[S] L. Schwartz, Théorie des distributions, Hermann, Paris, 1966. MR 35:730

[W] D. V. Widder, The heat equation, Academic Press, New York, 1975. MR 57:6840

Department of Mathematics, Sogang University, Seoul 121-742, Korea

E-mail address: sychung@ccs.sogang.ac.kr

Department of Mathematics, Seoul National University, Seoul 151-742, Korea

E-mail address: dohankim@snu.ac.kr

Department of Mathematics, Dongyang Technical College, Seoul 152-714, Korea

E-mail address: eglee@orient.dytc.ac.kr 